\title{
ARTICLE
}

\section{Preliminary study on precision dosimetry using radio-photoluminescent glass dosimeters for future use in radiotherapy}

\author{
Nazia Neelam Shehzadi ${ }^{\mathrm{a}, \mathrm{b}}$, Jae Pil Chung ${ }^{\mathrm{a}}$, In Jung Kimª, Byoung Chul Kimª and Chul-Young Yi ${ }^{\mathrm{a}, \mathrm{b}}$ * \\ ${ }^{a}$ Korea Research Institute of Standards and Science, 267 Gajeong-ro, Yuseong-gu, Daejeon 34113, Republic of Korea; ${ }^{b}$ University of \\ Science and Technology, 217 Gajeong-ro, Yuseong-gu, Daejeon 34113, Republic of Korea
}

\begin{abstract}
The radio-photoluminescence glass dosimetry test was carried out carefully. For the test, a therapy level Co-60 gamma-ray beam was used for the irradiation of the known doses to the dosimeters with the standard uncertainty of $0.7 \%(k=1)$. A weak negative correlation between mass and response of the glass dosimeter was found with Pearson's correlation coefficient $-0.27,-0.29,-0.29,-0.27$ and -0.26 for glass dosimeters irradiated at 1, 3, 5, 7 and 9 Gy radiation doses, respectively. It has been confirmed that the statistical uncertainty of the radio-photoluminescence glass dosimetry can be obtained within $1 \%$ from the repeatability test.
\end{abstract}

Keywords: radio-photoluminescence; passive dosimeter; repeatability; precision; linearity; dose; radiotherapy

\section{Introduction}

Radio-photoluminesent glass dosimeter (RPLGD) has attracted the attention in the medical physics society since the development of new automated reading system. This is a passive solid state dosimeter which is comparable to the different rival dosimeters such as thermoluminescence dosimeter (TLD) and optically stimulated luminescent dosimeter (OSLD).

A useful property of RPLGD is dose accumulation [1-6]. Radio-photoluminesent glass dosimeter (RPLGD) consists of phosphate glass $\left(\mathrm{PO}_{4}{ }^{3-}\right)$ doped with silver $\left(\mathrm{Ag}^{+}\right)$. Silver ions produce the colour centres or defects which turn into $\left(\mathrm{Ag}^{++}\right.$or $\left.\mathrm{Ag}^{\circ}\right)$ after irradiation. These defects are stable at room temperature and have capability to absorb and release radiation energy. By preheating the glass dosimeters, all the ions get enough energy to be stored in the radio-photoluminesent centres. The response may be inaccurate without preheating [7-9].

At first approximation, the magnitude of the response of a dosimeter may be directly proportional to its mass. In the case, the response variation of individual dosimeters can be reduced by normalizing the measured response to the dosimeter mass.

In principle, precision relates to better repeatability of the measurement and leads to lower uncertainty. The linearity of the dosimeter response against the irradiated dose is also important.

Previously, we reported the study of characteristics of

*Corresponding author. Email: cyyi@kriss.re.kr the RPLGD, in which we have presented that the response of the glass dosimeter was affected by number of ultra violet (UV) laser pulses, number of continuous repeatable readings, and the rotation and the position of glass dosimeters in the reading magazine. It was shown that the influences of neighbouring glass dosimeters and magazine slots were not negligible but by controlling these factors, the statistical uncertainty of the measurement could be reduced [10-11].

As a series of studies on RPLGD, the purpose of the present work is to test the feasibility of the precise radio-photoluminescent glass dosimetry by evaluating carefully the basic metrological properties of the measurement such as the mass-response relationship and repeatability.

\section{Materials and methods}

\subsection{Glass dosimeters and reading system}

FGD-1000 reader (Dose ACE), magazine (standard model, FGD-M151) and RPLGDs 302 M (Asahi Techno Glass) were used. The reader has UV laser pulse system for reading. RPLGDs $302 \mathrm{M}$ with Lot No. FD7130329-2 and FD731213-2 were used. These dosimeters have the dimension of $1.5 \mathrm{~mm} \times 12 \mathrm{~mm}$ [12]. All the glass dosimeters were annealed at $400{ }^{\circ} \mathrm{C}$ for 1 hour before irradiation.

\subsection{Irradiation setup}

For irradiation, a set of RPLGDs was loaded in an 
acrylic phantom $(300 \times 300 \times 100) \mathrm{mm}^{3}$, at $5 \mathrm{~g} / \mathrm{cm}^{2}$ water equivalent depth. The schematic diagram of irradiation setup is shown in Figure 1. The phantom was placed at $100 \mathrm{~cm}$ source-to-surface distance (SSD) from the $145 \mathrm{TBq}{ }^{60} \mathrm{Co}$ gamma-ray source installed in Korea Research Institute of Standards and Science (KRISS), where the field size was $(10 \times 10) \mathrm{cm}^{2}$. The phantom is designed in such a way that inner phantom can fit into the acrylic body of the phantom and inner jig can be inserted to fit in the inner phantom and glass dosimeters were replaced for each irradiation as shown in Figure 1. The standard uncertainty of the irradiated absorbed dose to the dosimeters by the $145 \mathrm{TBq}{ }^{60} \mathrm{Co}$ gamma-ray beam is evaluated to be $0.7 \%$ at $k=1$ combining, i.e., computing square root of summed square of, all uncertainties involved including those of the absorbed dose to water standard of $0.35 \%$ measured at the irradiation location with a calibrated ionization chamber and the dosimeter positioning of $0.58 \%$.

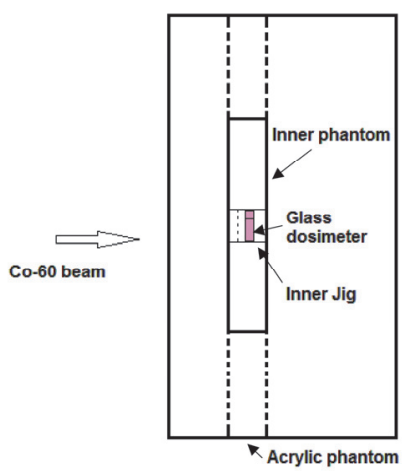

(a)

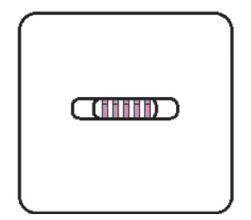

(b)

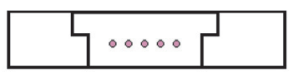

(c)
Figure 1. Schematic diagram of irradiation setup (a) Side view of acrylic phantom (b) Front view of inner phantom (c) upper view of inner phantom.

\subsection{Mass correlation}

60 RPLGDs from a batch were used for mass correlation study. Masses were measured using analytical balance yielding a mean value of $54.64 \mathrm{mg}$ $\pm 2 \mu \mathrm{g}$. The RPLGDs were annealed before irradiation. The responses were measured after irradiation. The procedure was applied repeatedly for radiation doses 1 , 3, 5, 7 and 9 Gy.

\subsection{Repeatability}

The repeatability was tested using 50 RPLGDs from a batch which were irradiated with 2 Gy. 5 readings were taken to observe the amount of deviation in readings. A reading was composed of 10 successive readouts and each readout value was produced by a different UV laser pulse triggered in the RPLGD reader. We tested two measurement schemes. Firstly, readings were taken continuously without changing any parameters. After completing first reading of all 50 RPLGDs, second reading was started up to complete 5 repeated readings of each RPLGD. Secondly, magazine was taken out of the reader after each reading and alternate 10 RPLGDs were placed inside the magazine for reading step by step. The procedure was also repeated 5 times.

\section{Results and discussion}

\subsection{Mass correlation}

The RPLGD responses were shown in Figure 2 as a function of their masses.

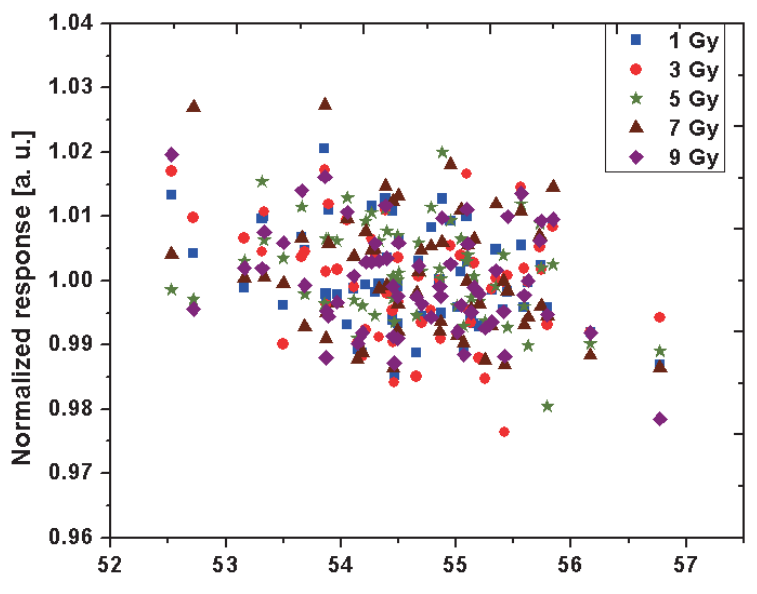

Mass of radio-photoluminescent glass dosimeter $(\mathrm{mg})$

Figure 2. RPLGD mass correlation with its response.

By definition, Pearson's correlation is used to analyse the strength and direction of the linear relationship between two continuous and normally distributed variables [13]. In our study, mass of RPLGD and their normalized response to specific radiation dose are variables of interest. We have checked the skewness value for each case of radiation dose and found that our variables are normally distributed as shown in Table 1. Pearson's correlation analysis was conducted between their masses and responses of RPLGDs for the irradiated doses 1, 3, 5, 7 and 9 Gy, respectively. Significance of results was also estimated by p-value to test the null hypothesis that there is no correlation. Table 1 shows the results of statistical test for the correlation between mass and response of RPLGD.

Table 1. Statistical analysis of mass correlation.

\begin{tabular}{cccc}
\hline $\begin{array}{c}\text { Irradiated } \\
\text { doses } \\
(\mathrm{Gy})\end{array}$ & Skewness & P- value & $\begin{array}{c}\text { Pearson's } \\
\text { correlation } \\
\text { coefficient }\end{array}$ \\
\hline 1 & 0.27 & 0.022 & -0.27 \\
3 & -0.08 & 0.028 & -0.29 \\
5 & -0.64 & 0.017 & -0.29 \\
7 & 0.56 & 0.039 & -0.27 \\
9 & 0.13 & 0.042 & -0.26 \\
\hline
\end{tabular}

Pearson's correlation coefficients between masses and responses of RPLGDs for all irradiated doses 1, 3, 5, 7 and 9 Gy indicate a weak negative correlation. If we 
consider p-value, the null hypothesis (i.e., no correlation) can be rejected with $95 \%$ level of confidence. On the whole, a weak but obvious negative mass-response correlation of RPLGD is observed. The rate of RPLGD response change estimated over the entire data in figure 2 is $0.2-0.4 \%$ per $\mathrm{mg}$ of its mass for our glass dosimeter model.

Evan's guide [14] was used for analysis of the strength and direction of the relationship, where the Pearson's correlation coefficients between 0.20 and 0.39 represent a 'weak association'.

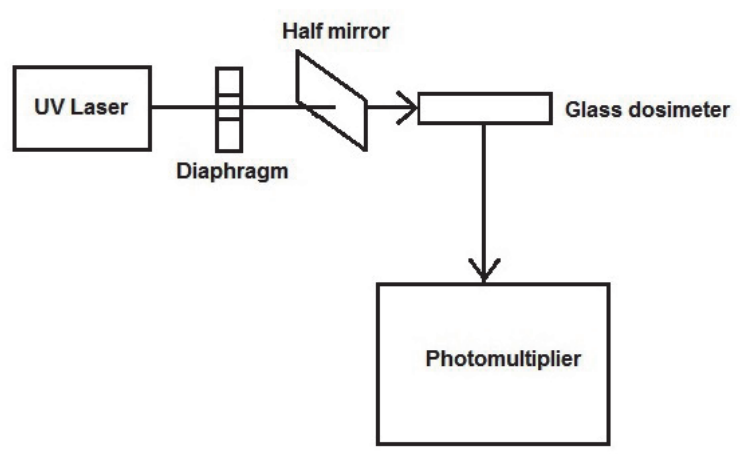

Figure 3. Simplified block diagram of RPLGD reader.

RPLGD is composed of $31.55 \% \mathrm{P}, 51.16 \% \mathrm{O}, 6.12 \%$ $\mathrm{Al}, \quad 11.00 \quad \% \quad \mathrm{Na}$ and $0.17 \% \quad \mathrm{Ag} \quad[1,3]$. The photoluminescence signal depends on the silver (Ag) concentration in the RPLGD mass. Ag concentration controls the absorption of UV laser beam and therefore the possible attenuation. Optical attenuation can be realized possibly by optical absorption. Also, in the path of light beam attenuation may be variable [15]. The simplified and comprehensive block diagram of RPLGD reader is shown in Figure 3.

\subsection{Repeatability}

As RPLGD has the property of non-destructive reading, a number of repeatable readings can be taken for an irradiated RPLGD. The statistical uncertainty associated with 5 repeated readings of each RPLGD is related to precision of measurement of an individual RPLGD.

In Figure 4, repeatability of RPLGD is shown, by two methods: 'continuous' with no disturbance and 'step by step' with possible disturbance during reading.

In the case of 'continuous' readings, no parameter was changed during reading and the coefficient of variation $(\mathrm{COV})$ for each RPLGD was evaluated. For analysing over 50 RPLGDs, COV was found ranging from $0.2 \%$ to $0.8 \%$.

But in case of 'step by step' reading, the magazine loaded with RPLGDs was taken out. The positions of the RPLGDs in the magazine were reset and put in the reader again after each reading. And then next reading was taken. We found that COV in the 'step by step' case has risen to $0.9 \%$ but it is still less than $1 \%$. We have controlled our measurement procedures according to our previous study about characteristics of RPLGD affecting their response [10].

The results for responses of all individual RPLGD were plotted against their ID number in Figure 4 where the error bars represent the standard uncertainty.

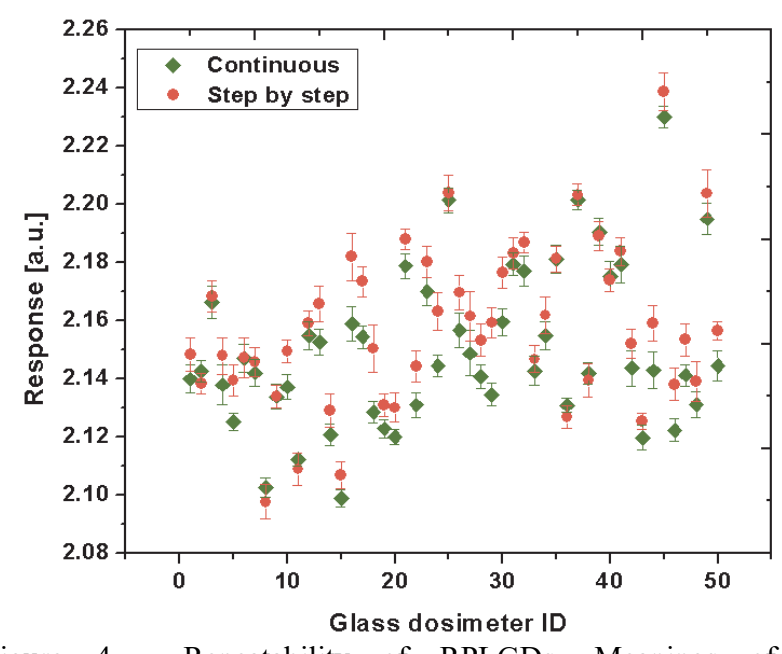

Figure 4. Repeatability of RPLGDs. Meanings of 'continuous' and 'step by step' are given in the text.

\section{Concluding remarks}

It was confirmed that the correlation between response of RPLGD and its mass was weak but evident from Pearson's correlation coefficient and p-value. A negative correlation was observed. The present test results of the repeatability along with that of the linearity of RPLGD response reported previously, [16] validated the concept of the precision dosimetry using RPLGD applicable to the radiation therapy dosimetry where an uncertainty of the dose measurement of a few percent is required.

In future, other characteristics of RPLGD like energy dependence, fading and dose rate dependence will be evaluated for setting up the precision dosimetry procedure for the radiotherapy applications including the external audit and the patient dose verification.

\section{Acknowledgements}

This work was supported by the Korea Research Institute of Standards and Science partly under the research project 'Development of Measurement Standards for Ionizing Radiation' with grant number 17011059 and in part by 'Expansion of Measurement Standard infrastructure for Medical use of Radiation' with the research project number 2014M2B8A3032605 granted by the Ministry of Science, ICT and Future Planning (MSIP)

\section{References}

[1] H. Mizuno, T. Kanai, Y. Kusano, S. Ko, M. Ono, 
A. Fukumura, K. Abe, K. Nishizawa, M. Shimbo, S. Sakata, S. Ishikura and H. Ikeda, Feasibility study of glass dosimeter postal dosimetry audit of high-energy radiotherapy photon beams, Radiother. Oncol. 86 (2008), pp. 258-263.

[2] F. Araki, N. Moribe, T. Shimonobou and Y. Yamashita, Dosimetric properties of radiophotoluminescent glass rod detector in high-energy photon beams from a linear accelerator and Cyber-Knife, Med. Phys. 31 (2004), pp. 1980-1986.

[3] J.-E. Rah, J.-Y. Hong, G.-Y. Kim, Y.-L. Kim, D.-O. Shin and T.-S. Suh, A comparison of dosimetric characteristics of a glass rod dosimeter and a thermoluminescent dosimeter for mailed dosimeter, Radiat. Meas. 44 (2009), pp. 18-22.

[4] Z. Knezevic, N. Beck, D. Milkovic, S. Miljanic and M.R. Komer, Characterisation of RPL and TL dosimetry systems and comparison in medical dosimetry applications, Radiat. Meas. 46 (2011), pp. 1582-1585.

[5] K. Son, H. Jung, S.H. Shin, H.-H. Lee, M.-S. Kim, Y.H. Ji and K.B. Kim, Evaluation of the dosimetric characteristics of a radiophotoluminescent glass dosimeter for high-energy photon and electron beams in the field of radiotherapy, Radiat. Meas. 46 (2011), pp. 1117-1122.

[6] W.K. Chung and D.W. Kim, Characteristic study of a radio-photoluminescence glass rod detector for clinical usages: skin and inner body in-vivo verification, J. Korean Phys. Soc. 62 (2013), pp. 670-676.

[7] D.Y.C. Huang and S.-M. Hsu, Radio-photoluminescence glass dosimeter (RPLGD), Advances in Cancer Therapy, (Prof. Hala Gali-Muhtasib), In Tech, Online, (2011), pp. 553-568, ISBN 978-953-307-703-1

[8] T. Yamamoto, RPL dosimetry: Principles and applications, AIP Conference Proceedings 1345 (2011), pp. 217-230.

[9] A.-L. Manninen, A. Koivula and M.T. Nieminen, The applicability of radiophotoluminescence dosimeter (RPLD) for measuring medical radiation (MR) doses, Radiat. Prot. Dosimet. 151 (2012), pp. 1-9.

[10]N.N. Shehzadi, J.P. Chung, B.C. Kim, I.J. Kim and C.-Y. Yi, Characteristics of radio-photoluminescent glass dosimeter reading system in radiotherapy applications, Proceedings of the Korean Association for Radiation Protection, Jeju, Korea, (2016), pp.352-353.

[11]K. Son, Y.H. Ji, K.B. Kim, M.S. Kim and H. Jung, The analysis of readout values according to reading method: Glass dosimeter reader, Radiat. Meas. 59 (2013), pp. 214-217.

[12]Chiyoda Technol Corporation, (2007), http://www.c-technol.co.jp/eng/e-small, RPL Glass Dosemeter/Small Element System Dose Ace DoseAceXe Technical Gide 2007, Asahi Glass Co., Ltd.

[13]M.M. Mukaka, Statistics corner: a guide to appropriate use of correlation in medical research, Malawi Medical Journal, 24(3) (2012), pp. 69-71.

[14]J.D. Evans, Straightforward statistics for the behavioral sciences, Pacific Grove, CA: Brooks/Cole Publishing (1996).

[15]Paschotta, article on 'optical attenuators' in the Encyclopedia of Laser Physics and Technology, 1. Edition October (2008), Wiley-VCH, ISBN 978-3-527-40828-3

[16]N.N. Shehzadi, J.P. Chung, B.C. Kim, I.J. Kim and C.-Y. Yi, Dosimetric characteristics of radio-photoluminescent glass dosimeters for medical applications: Linearity, Proceedings of the Korean Association for Radiation Protection, Gunsan, Korea, (2017), pp.370-371. 